\title{
2-D Electrothermal Simulation and Failure Analysis of GTO Turn-off with Complete Chopper Circuit Parasitics
}

\author{
Y. C. Gerstenmaier and H. Brunner \\ Corporate Research and Development, Siemens AG \\ Otto-Hahn-Ring 6, D-81739 München, GERMANY
}

\begin{abstract}
2-D electrothermal simulations of GTO-thyristor turn-off process including a complete chopper circuit and parasitic stray inductances will be presented. Turn-off failure is investigated for a single GTO cell and on a wafer scale using a homogeneous wafer, which has in parallel a one segment GTO representing a local perturbation on the wafer by a slightly different doping or carrier lifetime profile. During the spike voltage extreme power densities may result in the perturbed segment due to current filamentation. In high power GTO turnoff two destruction mechanisms have to be dealt with: 1.current-filamentation during the spike voltage period and 2.dynamic avalanche during the tail-phase.
\end{abstract}

\section{Simulation of Single GTO Cell and Wafer Scale GTO}

In this paper* for the first time 2-D electrothermal simulations of GTO-thyristor turnoff process including a complete chopper circuit and parasitic stray inductances will be presented. The device simulator MEDICI [1] solves the complete semiconductor equations (Poisson and continuity equations) together with the heat flow equation for the dynamic development of the lattice temperature within a general external network. Additionally thermal resistors and capacitors can be included in order to allow for the cooling of the device by the packaging.

In Fig. 1 the simulated circuit is shown with RCD-protection (snubber) circuit, inductive load, clamp and gate-drive circuit. Fig.2 displays the simulated GTO half cell with anode short-structure and Fig. 3 shows a simulation result for a $4.5 \mathrm{kV} / 3000 \mathrm{~A}$ GTO, that reveals the characteristics of measured results like storage time, spike voltage $\mathrm{U}_{D S P}$, peak off-state voltage $\mathrm{U}_{D M}$ and tail-current behaviour.

At the beginning of the turn-off process the GTO has a homogeneous temperature of $300^{\circ} \mathrm{K}$. The maximal local temperature of $316^{\circ} \mathrm{K}$ is attained during the spike voltage at the center junction in the middle under the cathode contact. As was already observed in the isothermal simulations of [2] the power density at that instant is very high, but according to our result not sufficient to cause a dramatic or dangerous temperature increase. In Fig. 4 the current density to be turned off is ten times larger. Now temperatures around the silicon melting point of $1700^{\circ} \mathrm{K}$ arise and would cause thermal destruction. The turn-off failure becomes apparent by the sudden anodevoltage breakdown in the tail-phase. Despite the negative gate-voltage and current

\footnotetext{
*This work was supported by eupec, a company of AEG and Siemens
} 
the device is not able to sustain the blocking voltage, because of the local loss of blocking capability at the center junction (Fig.5 and Fig.6). This is due to the fact that the temperature at the hot spot exceeds the intrinsic temperature of $550^{\circ} \mathrm{K}$ considerably.

Turn-off failure in the tail-phase has already been observed in isothermal 1-D simulation ([3] and references cited there) and 2-D simulation [4] and is due to dynamic avalanche generation, i.e. the steepening of the electric field gradient in the $n$-base by hole transport from the anode. In [4] turn-off failure was solely attributed to this. The measurements of [5] were done without snubber cicuit and clamp, so that there was no spike voltage. They revealed that a temperature maximum occurs just after the anode current fall time.

On a wafer scale many individual GTO-Segments $(>2000)$ in parallel contribute to current transport. Due to inhomogenities between different cells, current redistribution during turn-off takes place [6] (independently of dynamic avalanche) and may destroy those segments, which carry the most heavy load. For a special circuit without stray inductances and snubber circuit (no spike voltage) it was shown in [6], that with a gate current as high as the load current or higher the current filamentation can be damped but for usual gate drives this is not possible. [7] presented an analytical model in order to demonstrate, that during dynamic avalanche current filamentation may take place even for completely homogeneous devices.

The spike voltage, which arises during fall time, may cause destruction due to current filamentation. The current filaments in this case are not caused by dynamic avalanche as in [7] but, as we shall see, by slight inhomogenities in the lateral dopant or carrier lifetime distribution over the device area.

Because of the excessive number of grid-points it is not possible to simulate a whole GTO wafer. Therefore in Fig.7 the result is shown for a homogeneous $2.5 \mathrm{kV} / 2.5 \mathrm{kA}$ GTO (half cell scaled to full wafer size of $20 \mathrm{~cm}^{2}$ ), which has in parallel a one segment GTO $\left(0.024 \mathrm{~cm}^{2}\right.$ area) representing a local perturbation on the wafer by a slightly lower p-base doping of $10 \%$. Fig. 8 shows that precisely at the time of the spike voltage the one segment GTO takes over a maximum current 100 times larger than its on-state value. Since the maximum current of the small GTO segment exactly matches the time of the spike voltage, extreme power densities occur, which will lead to destruction. Somewhat lower current peaks are observed in the tail-period by dynamic avalanche, when the anode voltage is high. If avalanche generation in the parallel one segment GTO is turned off a smooth behaviour of the tail current emerges, whereas the current peak at spike voltage time is nearly the same. Therefore destruction at spike voltage time occurs independent of dynamic avalanche.

By suitable design the current redistribution can be reduced. In [8] similar isothermal simulations were carried out for 1-D devices in parallel; however without snubber circuit and spike voltage. Even though the p-base doping difference amounted to $28 \%$ the current redistribution between the cells was not dramatic.

\section{Conclusion}

The essential meaning of the spike voltage for GTO turn-off failure is revealed. Two destruction mechanisms have to be dealt with in high power GTO turn-off: 1 . currentfilamentation during the spike voltage period and 2. dynamic avalanche during the tail-phase. Both failure mechanisms can be investigated in numerical simulation with respect to design variations. 


\section{References}

[1] Technology Modeling Associates. Inc. Palo Alto, California, USA, MEDICI user's manual. March 1992.

[2] M. Turowski, A. Napieralski, J.M. Dorkel, Proceedings EPE-MADEP Firenze 1991, p.0-363-0-368.

[3] Y.C. Gerstenmaier, ISPS'92 Proceedings, 1992, Czech Technical University in Prague, p.49.

[4] I. Omura and A. Nakagawa, Proceedings of 1992 International Symposium on Power Semiconductor Devices \& ICs, Tokyo, pp.112-117.

[5] H. Ohashi and A. Nakagawa, IEEE IEDM-81 Tech. Dig., p.414, 1981.

[6] K. Lilja and H. Grüning, IEEE PESC Dig., p.398, 1990.

[7] G.K. Wachutka, IEEE Trans. on Electron Devices, Vol.38, p.1516, 1991.

[8] C.M. Johnson and P.R.Palmer, IEEE Trans. on Power Electronics, Vol.6, p.308, 1991.
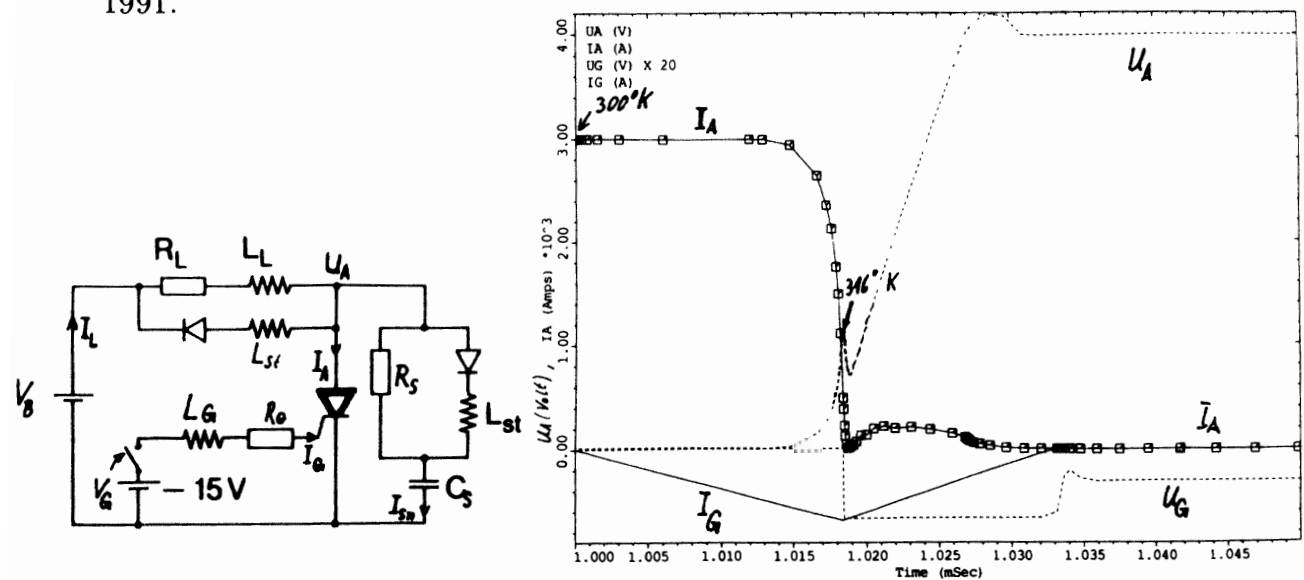

Figure 1: Simulated chopper-circuit for GTO turn-off.

Figure 3: 2-D electrothermal MEDICI simulation of GTO turn-off for circuit of Fig.1. Highest local temperature is marked at the spike voltage.
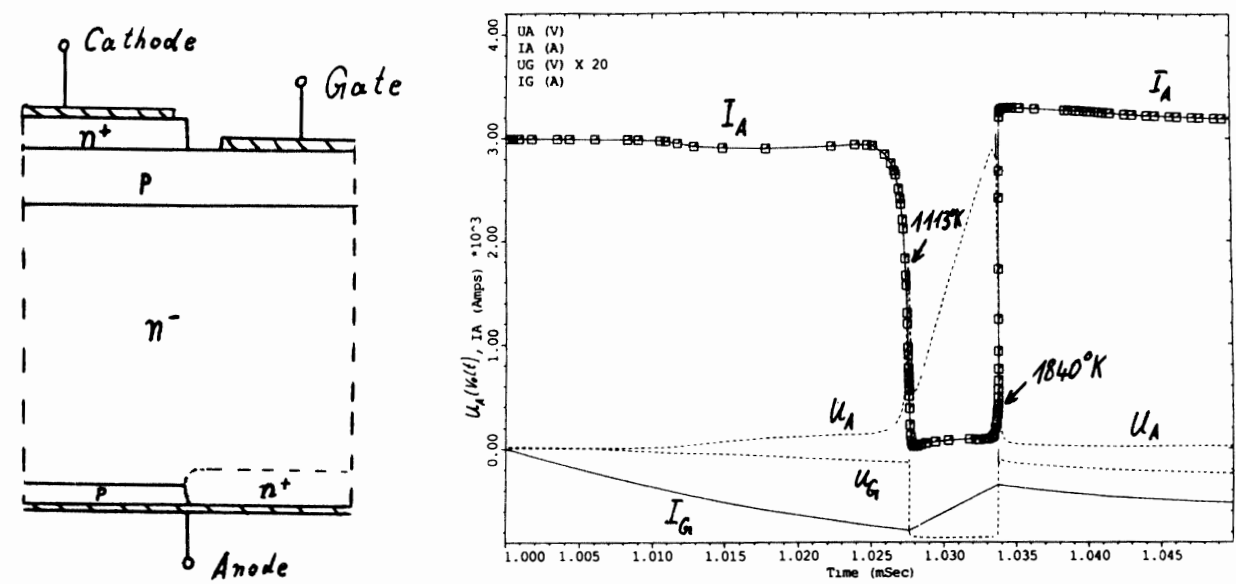

Figure 2: Simulated GTO half cell Figure 4: Destructive failure of GTO turn-off in 2-D with anode short structure. simulation at ten times the current density of Fig.3. 
56 Y. C. Gerstenmaier et al.: 2-D Electrothermal Simulation and Failure Analysis of GTO Turn-off
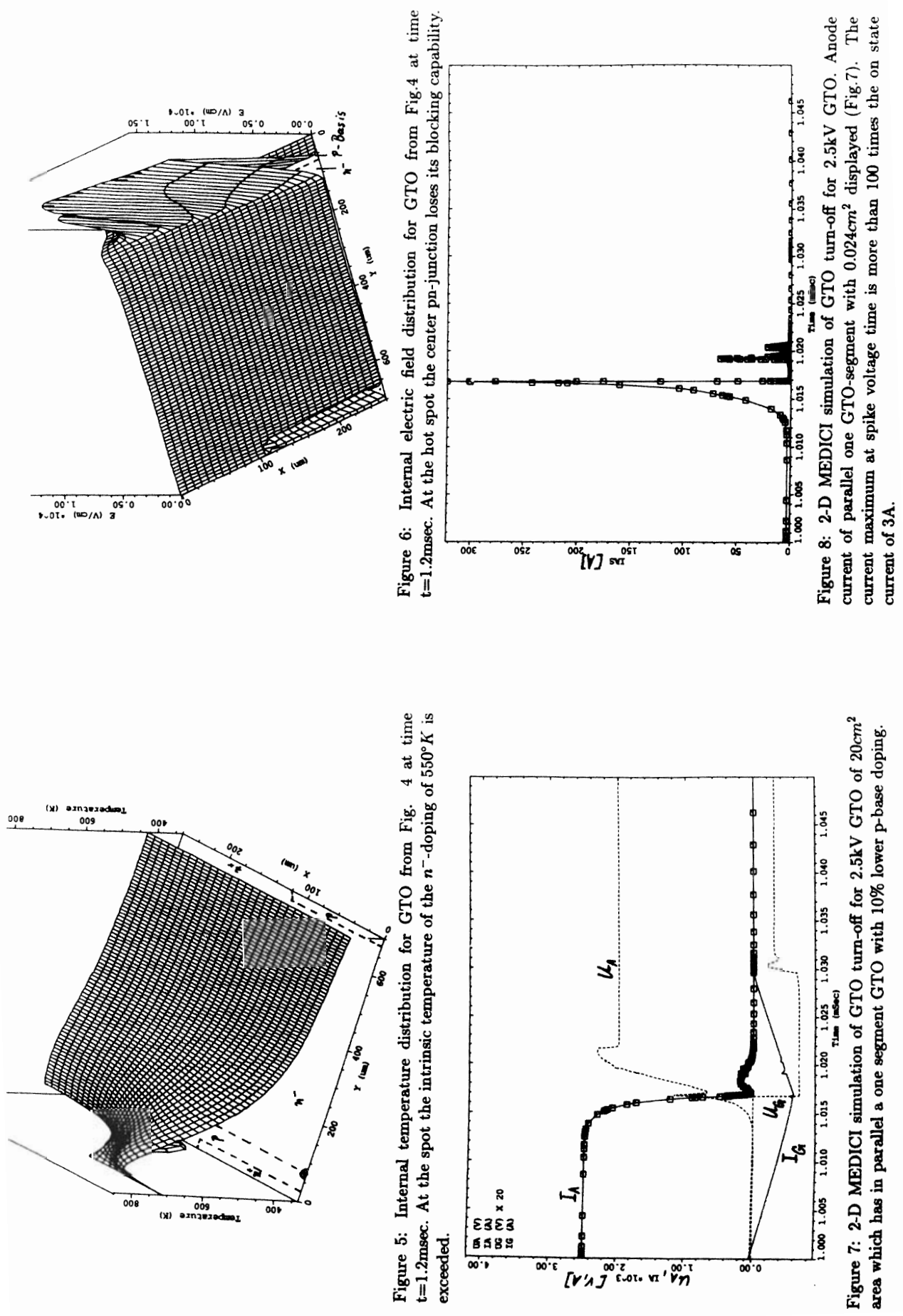